\title{
Enhancing Plasmonic Biosensors with Nanotechnology
}

\author{
Attila Bonyár \\ Department of Electronics Technology, Budapest University of Technology and Economics, \\ (BME-ETT), Budapest, Hungary \\ bonyar@ett.bme.hu
}

\begin{abstract}
The aim of the paper and the accompanying presentation is to give an introduction into biosensor technology, point out the current challenges and trends in their research and development and discuss through examples the possibility to apply nanotechnology to overcome these challenges. The paper reviews the utilization of nanomaterials to enhance the capabilities of optical (nanoplasmonic) sensors and collects the most widespread fabrication technologies of such nanomaterials. The possibility and challenges to integrate these sensors in to handheld point-of-care devices will also be discussed. The accompanying presentation is part of the Eurotraining course "Nanotechnology for electronics", held in the framework of the ISSE 2015 conference.
\end{abstract}

\section{INTRODUCTION}

Biosensors are analytical devices, which utilize biologically active molecules as sensing elements and are capable to measure the concentration of target molecules in even complex biological samples (blood, saliva, urine etc.) [1]. It is important to emphasize the utilization of biologically active molecules (sometimes common chemical sensors - such as a $\mathrm{pH}$ sensor - are incorrectly entitled biosensors when they are applied on biological samples), since they grant the special properties, most importantly the excellent selectivity for target molecules, which are characteristic to these sensors. Figure 1 presents the structural composition and working principle of biosensors in general. A biosensor can structurally be divided into two main, tightly connected parts. The bioreceptor (or biorecognition) layer consist of the biologically active molecules which interact with the tested sample and can specifically bind the target molecules (analyte). The function of the transducer is to convert the receptor-target molecular scale binding event (or reaction) into a measurable physical quantity, most commonly into an electrical or optical signal, which is subsequently processed by electronics to display the concentration data. The biomolecules are bound (immobilized) onto the surface of the transducer through a process called biofunctionalization, which is a critical step, since the biomolecules should retain their capability to form bonds with the target molecules, while attached to the surface.

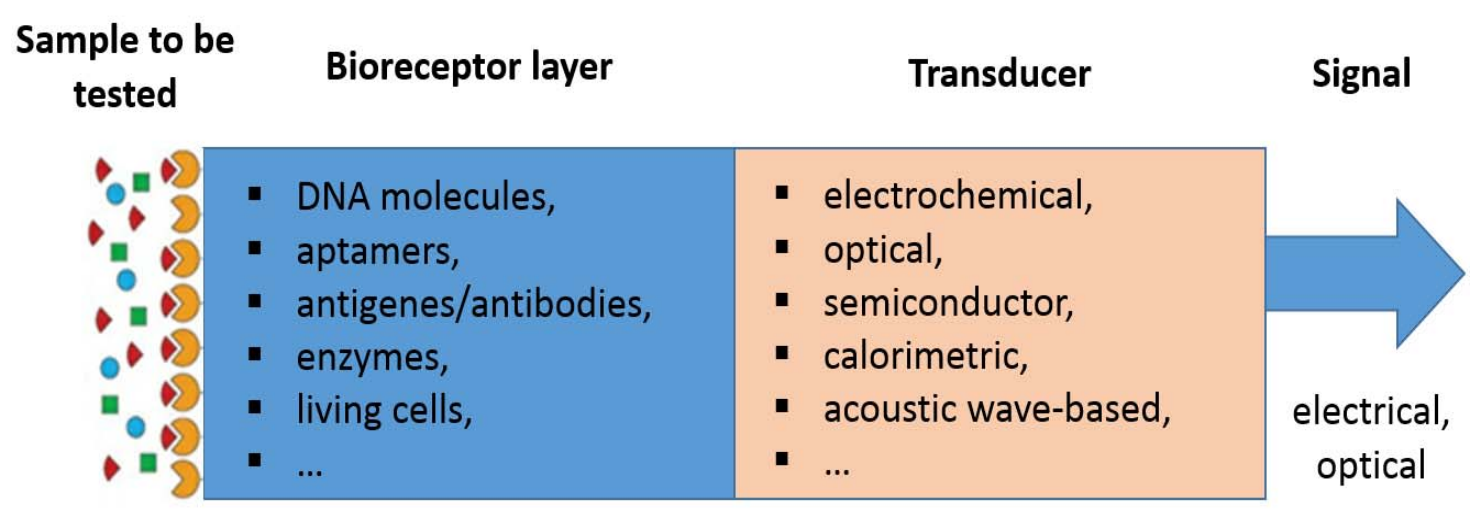

Fig. 1. Schematic illustration of the structural composition and working principle of biosensors in general. 
Based on the bioreceptor layer, the two main biosensor categories are the affinity type and reactive (catalytic) type sensors. The latter - currently more widespread on the market thanks to the success of the blood-glucose sensors - utilizes the biocatalytic properties of enzymes (enzymatic biosensors) or living cells (living biosesnsors) to selectively bind targets and detect their concentration by measuring the products of enzyme reaction or cell metabolism. In the other group of sensors the word affinity refers to the key-lock binding mechanisms, which can be found between these biomolecules and their respective pairs. This category could be divided into two sub-types: nucleotide sensors, which utilize the specific binding between two complementary single stranded ss-DNA chains and immunosensors, which use proteins e.g. antigenes and antibodies as sensing elements.

Before categorizing the biosensors by their transducers, we first have to clear the concept of labelfree sensing. In a classical DNA- or immune assay a fluorescent labeling molecule is attached to the target molecule usually prior the binding event, and a laser fluorescent microscope is subsequently used to evaluate the assay. Label-free biosensors however do not require such labels - which would increase the sample preparation time and cost - and their transducers directly transfer the binding event into measurable signal. Based on the transducer we can distinguish electrochemical sensors (using for example amperometry, voltammetry or electrochemical impedance spectroscopy (EIS) for evaluation); optical sensors (e.g. surface plasmon resonance (SPR), optical waveguide lightmode spectroscopy (OWLS)); sensors based on acoustic wave propagation (surface acoustic wave (SAW) and bulk acoustic wave (BAW) sensors, for example quartz crystal microbalance (QCM)); semiconductor based sensors (e.g. biofunctionalized field effect transistors (bio-FET)); calorimetric sensors (mostly used with enzymatic sensors).

The possible application of biosensors cover the fields of disease diagnostics, environmental monitoring (e.g. detection of viruses, bacteria, toxins or heavy metal ions in drinking water), food monitoring (e.g. detection of pesticides, toxins), gene analysis, biomarker detection, pharmaceutical research etc.

The concept of multi-biosensors refers to the detection of multiple target molecules with only one sensor element integrated into a chip or a cartridge. Combined with label-free sensing, only a couple of transduction techniques can enable this possibility. In the cases of electrochemical and FET bases sensors, the transducers need to address the different bioreceptor layers (multiple electrode pairs or transistors). In the case of SPR the so called imaging possibility (SPRi) enables the system to measure on a relatively large $\left(\mathrm{few} \mathrm{cm}^{2}\right.$ ) sensor area in even several hundred differently funcionalized spots in a parallel way.

Point-of-care (POC) devices are small medical instrument which enable the testing of the patient at or near the site of care. The most well-known POC devices are for blood or urine tests (e.g. to measure blood glucose concentration), but electrochemical biosensors are being developed for POC cancer diagnostics as well [2].

The most important sensor parameters considering the possible applications are the following. The limit of detection (LOD) could be critical for the applications, where the amount of detectable target molecules are very low in the sample (e.g. detection of pathogens from drinking water [3]). The sensitivity of the sensor could be important, when the relevant concentration range of the measured analyte is not extremely small, but its concentrations needs to be determined with high resolution. The stability and life time of the sensor is very important for point-of-care applications. The reproducibility of the sensor demands robustness from the fabrication technologies (especially when utilizing complex procedures). For some applications the regeneration of the receptor surface could be considered, to enable multiple measurements with the same sensor, but most of the point-of-care diagnostic devices count on disposable sensor chips/cartridges. In connection with the above mentioned points, the fabrication cost is also an important factor, which usually requires a compromise to match the needs (and possibilities) of the market.

In the following chapters the possibility to utilize nanomaterials in order to enhance these most important sensor parameters will be discussed for optical biosensors. Although there are several optical methods available for biosensing purposes, due to paper size limitations we will solely focus on surface plasmon resonance (SPR) and its nanotechnology aspects (nanoplamonics). 


\section{SPR PRINCIPLE}

Surface plasmon polaritons (SPPs) are the collective oscillation of delocalized electrons at a metallic surface in response to an external electric field (light). In a classical SPR refractive index sensing measurement setup usually a thin film of metal is used. To incite plasmons in metallic thin films special illumination conditions have to be met, so usually a reflective optical setup with a prism and other additional optics is utilized (often referred to as Kretschmann or Otto configuration). The intensity of the reflected light from the thin film decreases when the plasmons are excited, this intensity is depending on the following parameters: the wavelength of the incoming light $(\omega)$, the angle of incidence $(\varphi)$, the properties of the metallic layer, namely the electron density function $\left(N_{e}\right)$ and the layer thickness $(d)$, and finally the dielectric properties of the surrounding medium, characterized by the refractive index $(n)$. Based on this relation several detection methods are available using wavelength, angular or intensity detection using Kretschmann geometry (Fig. 2/a). Considering the metal layer, gold or silver are popular solutions since their plasmon absorbance bands are in the visible light region $[4,5]$.

The two major advantages of SPR sensors are their excellent sensitivity (even in the range of $10^{-7} \mathrm{RIU}$ [6]), and the fact that they are only sensitive to the changes in the refractive index of the media close to the metal-dielectric interface. The incited electric near field has intensity maximum at the interface and its exponentially decaying so called 'evanescent' region only penetrates the surrounding media for only the fraction of the wavelength of the incoming light usually a few $100 \mathrm{~nm}$ deep. This is exactly the region where our molecular scale interactions take place. Besides, SPR yields real-time information regarding the molecular binding events, which is a great advantage compared to the end-point systems. Also, by using a defocused laser illumination and a CCD camera as a detector it is possible to image a larger area of the sensor surface for the detection of several targets in a multi-biosensor concept (SPR imaging).

\section{LSPR PRINCIPLE}

When the size of a metallic nanoparticle is much smaller than the wavelength of the incoming light it is possible to excite the conduction electrons, which start to oscillate against the Coulomb forces of the core.
These incited plasmons are localized on the nanoparticle - unlike the propagating plasmons at a thin film dielectric interface in the case of classic SPR.

The biggest difference between LSPR and classic SPR is that localized surface plasmon resonance on a nanoparticle is always excitable, the problem of coupling the light into a thin film with suitable optics (prism, filters etc.) can be eliminated, and much simpler and more convenient measurement configurations can be used. Figure 2 compares the most widespread LSPR setups with the classical Kretschmann geometry (Fig. 2/a).

The simplest way is to use to nanoparticles for LSPR sensing in a colloidal form (Fig. 2/b) [7]. In this form the nanoparticles are free to interact with the samples to be tested, but a drawback is that the transmissive setup measures through the whole sample, so the bulk effect will not be eliminated (like in classic SPR). The same is true for the chip based LSPR setup (Fig. 2/c), where the nanoparticles are immobilized onto a suitable transparent substrate [8]. This chip can be used with corresponding microfluidics sample handling setups much like the classis SPRi chips, but in a significantly simpler transmissive optical configuration, which would enable the integration of this principle into small, handheld point-of-care devices. The third, optical fiber based LSPR setup utilized nanoparticles as a coating on optical fibers [7,9]. The transmitted light is reflecting from this special 'cladding' and thus it interacts with the nanoparticles. The advantage of this setup is that it is directly compatible with most of the spectrometers which use fiber probes.

\section{LSPR PERSPECTIVES AND CHALLENGES}

It can be seen, that due to the easier excitation of localized plasmons in nanoparticles LSPR enables simpler measurement configurations which can be integrated into miniaturized devices. However, we have to mention some challenges, which are needed to be optimized when designing an LSPR instrument. First of all, it is well known that the sensitivity of an LSPR setup - which is defined as the shift of the plasmon absorbance peak due to the refractive index change of the medium [nm/RIU] - is strongly depending on the size, shape and distribution of the applied nanoparticles. In a recent review M.H. Tu et al. collected the reported LSPR sensitivity values for 
several nanostructures [10]. The reported sensitivity values show great variation: from the simplest colloidal gold spheres $(71 \mathrm{~nm} / \mathrm{RIU})$ to the more exotic

gold nanocages (783-1933 nm/RIU) the difference can be more than one order of magnitude.

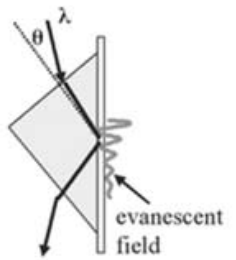

a)

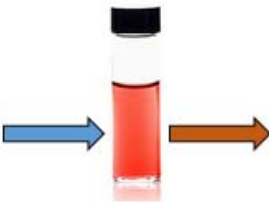

b)

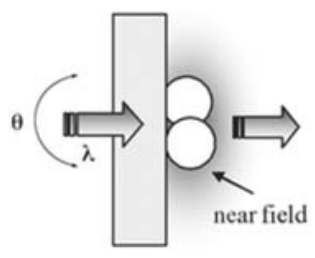

c)

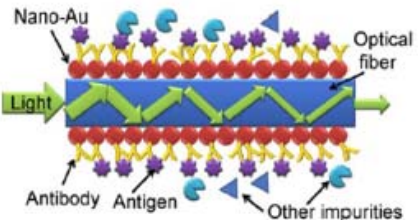

d)

Fig. 2. Schematic illustration of SPR and LSPR measurement configurations. a) classic Kretschmann SPR setup, b) colloidal LSPR setup, c) chip based LSPR setup, d) optical fiber based LSPR setup. Images reproduced from [7].

We have to note that, firstly this is bulk medium refractive index sensitivity, which could differ from molecular scale interaction sensitivity (on the particle surface) and secondly, that these sensitivity values are also depending on the measurement configuration. If a nanoparticle is fixed to the surface of a substrate (Fig 2c and d) a significant part of its near field will be filled with the fixed refractive index of the substrate material, thus its potential sensitivity could decrease.

Although the reported bulk sensitivity for thin film based classical SPR setups are currently well above the LSPR (it can be above $3300 \mathrm{~nm} / \mathrm{RIU}$ [11]), for the monitoring of molecular scale interactions close to the metallic surface the situation is different. $\mathrm{Xu}$ et al. studied the plasmonic behavior of gold nanoparticles fixed to glass substrates and found that excited near field decay is depending on the particle size [12]. The proposed $\left(r_{0} / r\right)^{6}$ decay length (where $r_{0}$ is the radius of the spherical nanoparticle and $r$ is the distance) is at least one order of magnitude smaller than the exponentially decaying evanescent field length in the case of thin film SPR. In other words, by using LSPR we are even more focused on the changes which take place in the near vicinity of the particle surface, and this is the region, where our molecular scale interactions occur in affinity type biosensors. It was already proven experimentally, that for molecular interactions - using biotin-streptavidin affinity bonding-the sensitivity of LSPR can match a standard thin film based SPR [11].

In this sense bulk sensitivity and field decay length must be taken account together, and the particle shape, size and distribution must be optimized according to the desired application for LSPR sensors. Thus, a suitable nanofabrication technology must be selected taking into account the following goals, which summarize all the advantages of classic SPR and LSPR: the integration of the LSPR principle into small, handheld point-of-care biosensor devices...

- without losing the excellent sensitivity of classic SPR,

- without losing the possibility of imaging, which means a large number of parallel measurements on the sensor surface,

- with cost effective nanofabrication technologies which could produce affordable disposable sensor chips/cartridges.

\section{LSPR NANOFABRICATION TECHNOLOGIES}

This chapter will collect the most relevant nanotechnologies which can be used for the fabrication of LSPR sensors and also compare their advantages/disadvantages considering the above mentioned goals.

\subsection{Electron/Ion beam lithography}

By utilizing the approximately $10 \mathrm{~nm}$ resolution of electron beam lithography (EBL) it is possible to design and fabricate an array of well oriented nanoparticles on a chip surface [13]. Besides the classical resist based technology, direct ion beam lithography (milling) and its variants (like gas assisted deposition or gas assisted etching) can also be used for this purpose [14]. The biggest concern of this technology is that the patterning of larger areas (which is required for SPR imaging) can be both problematic (only newer microscopes have adequate solutions for stitching the written areas) and time consuming which 
could increase the fabrication cost of the resulting sensor chip well above the desired affordable range.

\subsection{Nanoimprint lithography}

The general idea of nanoimprint lithography is to combine the precision and resolution of EBL with large area patterning capabilities of transfer technologies (replication). In the case of softnanoimprint first a master is formed in a photoresist with standard EBL technology, which pattern is later transferred into a soft stamping material (e.g. polydimethylsiloxane, PDMS). This master can subsequently (and repeatedly) be used as a molding form to fabricate an array of nanoholes or nanodots [15]. Although the initial master is still fabricated by EBL or IBL, the total fabrication cost can be somewhat lower (considering the multiple use of the stamp), and we still have the possibility to design the shape, size and distribution (e.g. spacing) of the nanostructures in the array.

\subsection{Colloid and surface chemistry}

Perhaps the most widespread technology for the production of nanoparticles is the controlled chemical synthesis or colloid chemistry. In this case the metallic nanoparticles are formed by reducing their precursor (which can be a dissolved salt of the metal) in a solution. A typical example is the controlled reduction of gold chloride (chloroauric acid, $\mathrm{HAuCl}_{4}$ ) in aqueous solution with citrate as a reducing agent. By controlling the molar ratio of the chloride and the reducing agent the size of the resulting spherical nanoparticles can easily be controlled (size controlled growth). In more complex systems the shape of the resulting particle can also be defined (shape controlled growth) and more complex compact (nanosphere, nanorod, nanocube, nanodisc etc.) or hollow (nanoshell, nanocage, etc.) particles can be produced [16].

Besides the cost (both the readily synthetized nanoparticles and their precursors/reagents are quite costly) the other disadvantage of the method is that the produced nanoparticles are needed to be fixed on a transducer surface, which can be quite challenging. There are techniques based on e.g. the silanization of glass (by using 3-aminopropyltriethoxysilane, APTES, as a linker to bind gold NP-s on the surface of glass) [17], or on thiol chemistry [18]. In both of the cases the biggest challenge is keeping the nanoparticles from aggregation and even if succeeding in this regards, the control on the resulting distribution of the nanoparticle array is limited (compared to lithography).

\subsection{Thermal annealing of thin films}

Perhaps one of the simplest and cheapest methods for the generation of metallic nanoparticles on a substrate is the thermal annealing of a thin layer of metal $(5-15 \mathrm{~nm})$ pre-deposited by vacuum technology (sputtering or evaporation) [19]. By controlling the technological parameters of the deposition and annealing process (initial layer thickness, annealing temperature, annealing time etc.) we can have some influence on the resulting size and distribution of the particles, but it is still limited compared to lithography. Another problem is that the resulting particles usually bind poorly to the substrate (e.g. gold to glass), so the subsequent fixation or transfer of the nanoparticles to another substrate have to be solved prior any application containing fluidic systems, which could remove the nanoparticles [20].

A possible solution to the first problem would be the application of nanopatterned substrates for the annealing. It is possible to create well-ordered pores e.g. by the anodic oxidation and subsequent etching of alumina-alumina-oxide systems, where the pore size can be controlled by the etchant and the temperature during anodization in a large range (approx. $20-90 \mathrm{~nm}$ ) [21, 22]. Such pores would behave as 'nests' during the annealing procedure, and define the position of the resulting nanoparticles, whose size can also be controlled by the initial layer thickness of the deposited metal. It can clearly be seen on Fig 3. that both the particle size and distribution is better controlled compared to the nanoparticles created on glass substrates.

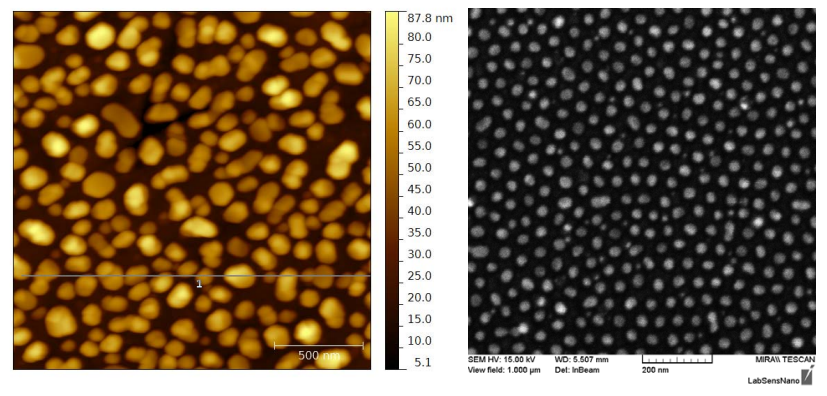

Fig. 3. Left: Tapping mode AFM image of thermally generated Au NPs (scan size $2 \times 2 \mu \mathrm{m}^{2}, \mathrm{Z}$ scale $87.8 \mathrm{~nm}$, own image). Right: SEM image of thermally generated Au NPs on porous alumina substrate (scalebar $200 \mathrm{~nm}$ ) [22]. 


\section{Conclusions}

Nanomaterials and LSPR offer a great possibility to integrate the versatile SPR principle into small point-of care devices - without losing its excellent sensitivity or imaging capability - due to its much simpler measurement configurations. However, the sensitivity of the sensor is strongly depending on the applied nanoparticles, so the selection of a suitable fabrication technology to optimize the cost-sensitivity tradeoff is extremely important considering the desired application. Future development in the fabrication technologies would enable the mass production of reliable and cheap LSPR chips for point-of-care applications.

\section{ACKNOWLEDGEMENTS}

This research was supported by the European Union and the State of Hungary, co-financed by the European Social Fund in the framework of TÁMOP 4.2.4. A/2-11-1-2012-0001 'National Excellence Program'. The author is grateful for the support of Bolyai János Scholarship.

\section{REFERENCES}

[1] Gábor Harsányi, "Sensors in biomedical applications: fundamentals, technology and applications", CRC Press, 2010.

[2] Celine I.L. Justino, Teresa A.P. Rocha-Santos, Armando C. Duarte, "Advances in point-of-care technologies with biosensors based on carbon nanotubes", TrAC Trends in Analytical Chemistry, Vol. 45, April 2013, pp. 24-36.

[3] H. Sántha, "Sustainable DNA/RNA release methods for in-line waterborne pathogen screening devices", In: G. Zuccheri, N. Asproulis, "Detection of pathogens in water using micro and nano-technology", IWA Publishing, 2012.

[4] J. Homola, S.S. Yee, G. Gauglitz, "Surface plasmon resonance sensors: review", Sensors and Actuators B: Chemical 54, 1999, pp. 3-15.

[5] Alexandre Dmitriev, "Nanoplasmonis Sensors", Springer, 2012.

[6] J. Homola, "Surface Plasmon Resonance Sensors for Detection of Chemical and Biological Species", Chem. Rev. 108, 2008, pp. 462-493.

[7] Jie Cao, Tong Sun, Kenneth T.V. Grattan, "Gold nanorod-based localized surface plasmon resonance biosensors: A review", Sensors and Actuators B: Chemical, Vo. 195, 2014, pp. 332-351.
[8] Borja Sepúlveda, Paula C. Angelomé, Laura M. Lechuga, Luis M. Liz-Marzán, "LSPR-based nanobiosensors", Nanotoday, Vol. 4, Issue 3, June 2009, pp. 244-251.

[9] M.H. Tu, T. Sun, K.T.V. Grattan, "Optimization of gold-nanoparticle-based optical fibre surface plasmon resonance (SPR)-based sensors", Sensors and Actuators B: Chemical, Vol. 164, Issue 1, March 2012, pp 43-53.

[10] M.H. Tu, T. Sun, K.T.V. Grattan, "LSPR optical fibre sensors based on hollow gold nanostructures", Sensors and Actuators B: Chemical, Vol. 191, February 2014, pp. 37-44.

[11] Svedendahl M, et al."Refractometric sensing using propagating versus localized surface plasmons: a direct comparison”, Nano Lett. 9 (12), 2009, pp. 4428-4433.

[12] Xu HX, Kall M. "Modeling the optical response of nanoparticle-based surface plasmon resonance sensors." Sens Actuators B Chem. 87 (2), 2002; pp. 244-249.

[13] Yifang Chen, "Nanofabrication by electron beam lithography and its applications: A review", Microelectronic Engineering, Vol. 135, March 2015, pp. 57-72.

[14] Shaoli Zhu, Wei Zhou, "Plasmonic properties of twodimensional metallic nanoholes fabricated by focused ion beam lithography", Journal of Nanoparticle Research 14, February 2012, pp. 652-658.

[15] Chen-Chieh Yu, Hsuen-Li Chen, "Nanoimprint technology for patterning functional materials and its applications", Microelectronic Engineering, Vol. 132, 25 January 2015, pp. 98-119.

[16] Dung The Nguyen, Dong-Joo Kim, Kyo-Seon Kim, "Controlled synthesis and biomolecular probe application of gold nanoparticles", Micron, Vol. 42, Issue 3, April 2011, pp. 207-227.

[17]Nicola E. Cant, Kevin Critchley, Hao-Li Zhang, Stephen D. Evans, "Surface functionalisation for the self-assembly of nanoparticle/polymer multilayer films", Thin Solid Films 426, 2003, pp. 31-39.

[18] Jozef Magura, Adriana Zeleňáková, Vladimír Zeleňák, Maria Kaňuchová, "Thiol-modified gold nanoparticles deposited on silica support using dip coating", Applied Surface Science, Vol. 315, October 2014, pp. 392-399.

[19] A. Serrano, O. Rodríguez de la Fuente, M. A. García, "Extended and localized surface plasmons in annealed $\mathrm{Au}$ films on glass substrates", Journal of Applied Physics, Vol. 108, 2010, pp. 074303.

[20] Tanya Karakouz, Ben M. Maoz, Gilad Lando, Alexander Vaskevich, and Israel Rubinstein, "Stabilization of Gold Nanoparticle Films on Glass by Thermal Embedding", Appl. Mater. Interfaces, 2011, 3 (4), pp. 978-987. 
[21] L. Zaraska, W. J. St, epniowski, E. Ciepiela, and G. D. Sulka, "The effect of anodizing temperature on structural features and hexagonal arrangement of nanopores in alumina synthesized by two-step anodizing in oxalic acid," Thin Solid Films, Vol. 534, 2013, pp. 155-161.
[22] Tomás Lednicky, "Template assisted deposition of multilayer nanostructures”, Master Thesis, 2014, Brno. 